\title{
A THEOREM ON CONNECTED GRAPHS IN WHICH EVERY EDGE BELONGS TO A 1-FACTOR
}

\author{
CHARLES H. C. LITTLE
}

(Received 27 April, 1973)

Communicated by G. E. Wall

\section{Introduction}

In this paper, we consider factor covered graphs, which are defined basically as connected graphs in which every edge belongs to a 1 -factor. The main theorem is that for any two edges $e$ and $e^{\prime}$ of a factor covered graph, there is a cycle $C$ passing through $e$ and $e^{\prime}$ such that the edge set of $C$ is the symmetric difference of two 1-factors.

\section{Definitions}

We adopt the definitions and notation of [1] with the following exceptions and additions. All graphs considered are connected. Let $G$ be a graph, and let $e=\{v, w\}$ where $e \in E(G)$ and $v, w \in V(G)$. It follows that $G-e=G-\{v, w\}$. The contradictory definition of $G-e$ given in [1] is not used in this paper. We call $v$ and $w$ the ends of $e$. Cycles, paths and 1-factors are defined as in [1] except that they are each regarded as sets of edges rather than sequences or subgraphs. If $C$ is a cycle, $V(C)$ is defined as the union of the edges of $C$. If $P$ is a $v-w$ path, we call $v$ and $w$ the terminal vertices of $P$.

The symmetric difference $F_{1} \Delta F_{2}$ of distinct 1-factors $F_{1}$ and $F_{2}$ is a set of disjoint cycles; these we call alternating cycles. Thus an alternating cycle can also be defined as a cycle $A$ of even length such that $G-V(A)$ has a 1-factor.

A graph with at least four vertices is called factor covered if for any edge there is a 1 -factor that contains that edge. Now let $e$ be an arbitrary edge of the factor covered graph $G$. Since $G$ is connected and $|V(G)| \geqq 4$, there is another edge incident on one of the ends of $e$, and this edge must belong to some 1-factor $F_{1}$. Therefore $e \notin F_{1}$. On the other hand $e$ belongs to some 1-factor $F_{2}$. Therefore $e \in F_{1} \Delta F_{2}$, and $e$ thus belongs to some alternating cycle of $G$. On the other hand, any edge that belongs to an alternating cycle certainly belongs to a 1-factor, and so we see that factor covered graphs are those connected graphs in which for any edge there is an alternating cycle containing that edge. 
If $A$ and $B$ are distinct subsets of $E(G)$, we define an $A B$-path to be a path $P$ of maximal length such that $P \subseteq A \cap B$. An $\bar{A} B$-path is defined as a path $P$ of maximal length such that $P \subseteq B-A$.

\section{The theorem}

We now prove that for any pair of edges of a factor covered graph, there is an alternating cycle containing those edges.

The proof of the theorem hinges on the following lemma. The author is indebted to P. W. Kasteleyn for greatly simplifying the original proof of this lemma.

LEMMA. Let $G$ be a connected graph with distinct 1-factors $F_{0}, F_{1}, F_{2}$ and alternating cycles $A_{1}, A_{2}$ such that $A_{1}=F_{0} \Delta F_{1}, A_{2}=F_{0} \Delta F_{2}$ and $E(G)=A_{1} \cup A_{2}$.

Then for any pair $e, e^{\prime}$ of edges of $G$ there is an alternating cycle containing both $e$ and $e^{\prime}$.

Proof. The proof is by induction on the number $n$ of $\bar{A}_{1} A_{2}$-paths in $G$.

Suppose $n=1$. If $e$ and $e^{\prime}$ are both in $A_{1}$ or both in $A_{2}$, the lemma is immediate; suppose therefore that $e \in A_{2}-A_{1}$ and $e^{\prime} \in A_{1}-A_{2}$. Then $F_{1} \Delta F_{2}$ is clearly an alternating cycle containing $e$ and $e^{\prime}$.

Now let the lemma be true whenever the number of $\bar{A}_{1} A_{2}$-paths is less than $n$. Again, if $e$ and $e^{\prime}$ are both in $A_{1}$ or both in $A_{2}$, the lemma is immediate; hence without loss of generality, let $e \in A_{2}-A_{1}$ and $e^{\prime} \in A_{1}-A_{2}$.

Clearly $A_{1} \Delta A_{2}=F_{1} \Delta F_{2}$, and $F_{1} \Delta F_{2}$ is the union of a collection of disjoint alternating cycles; let $C$ be the cycle in this collection which contains $e$. If $e^{\prime} \in C$, the proof is complete. Suppose therefore $e^{\prime} \notin C$.

Since $e^{\prime} \in A_{1} \Delta A_{2}$, we have $e^{\prime} \in F_{1} \Delta F_{2}$. Hence $e^{\prime}$ belongs to some alternating cycle $C^{\prime}$ which is a subset of $F_{1} \Delta F_{2}$. If $P$ is the $A_{2} A_{1}$-path that contains $e^{\prime}$, there must clearly be edges of $A_{2} \cap A_{1}$ (and therefore edges of $A_{2}-A_{1}$ ) incident on the terminal vertices of $P$ because of the maximality property of $\bar{A}_{2} A_{1}$-paths. The edges of $A_{2}-A_{1}$ incident on a terminal vertex of $P$ must belong to $C^{\prime}$ and therefore are not in $C$ since $C$ and $C^{\prime}$ are disjoint; hence $A_{2}-A_{1}-C \neq \varnothing$. It clearly follows that there exist $\bar{A}_{1} A_{2}$-paths whose edge sets are contained in $E(G)-C$. Let $G^{\prime}$ be the graph obtained from $G$ by deleting all non-terminal vertices of every such path.

Clearly $A_{1}$ and $C$ are alternating cycles of $G^{\prime}$ since $F_{1} \cap\left(C-A_{1}\right)$ and $F_{1} \cap\left(A_{1}-C\right)$ are 1-factors of $G^{\prime}-V\left(A_{1}\right)$ and $G^{\prime}-V(C)$ respectively. Furthermore, if for $i=0,1$ we define $F_{i}^{\prime}=F_{i} \cap E\left(G^{\prime}\right)$, then $F_{i}^{\prime}$ is clearly a 1-factor of $G^{\prime}$. In $G^{\prime}$, we have $A_{1}=F_{1}^{\prime} \Delta F_{0}^{\prime}$, and since $C=F_{1}^{\prime} \Delta F_{2}{ }^{\prime}$ for some 1-factor $F_{2}{ }^{\prime}$ of $G^{\prime}$, the hypotheses of the lemma hold for alternating cycles $A_{1}$ and $C$ in 
$G^{\prime}$. Since the number of $\bar{A}_{1} C$-paths in $G^{\prime}$ is less than $n$, the lemma must hold for $G^{\prime}$ by rhe induction hypothesis. Therefore there is an alternating cycle $A$ of $G^{\prime}$ that contains both $e$ and $e^{\prime}$.

Since $A$ is an alternating cycle, $G^{\prime}-V(A)$ has a 1 -factor, $F$ say. It follows that $F \cup\left(F_{0} \cap\left(E(G)-E\left(G^{\prime}\right)\right)\right)$ is a 1 -factor of $G-V(A)$. Therefore $A$ is an alternating cycle of $G$ containing $e$ and $e^{\prime}$, and the lemma is proved.

We are now ready to prove the theorem.

THEOREM. A graph $G$ is factor covered if and only if for every $e, e^{\prime} \in E(G)$ there exists an alternating cycle containing both $e$ and $e^{\prime}$.

Proof. The sufficiency of the given condition is clear, since any edge that belongs to an alternating cycle belongs to a 1-factor.

To prove necessity, let $P$ be the shortest path in $G$ containing both $e$ and $e^{\prime}$. $P$ exists because $G$ is connected. The proof is by induction on $|P|$. If $|P|=1$, then $e=e^{\prime}$ and the theorem follows from the fact that $G$ is factor covered.

If $|P|=2$, then $e$ and $e^{\prime}$ are both incident on some vertex $v$. Let $F$ and $F^{\prime}$ be 1-factors such that $e \in F$ and $e^{\prime} \in F^{\prime}$. Hence $e \notin F^{\prime}$ and $e^{\prime} \notin F$, so that $F^{\prime} \Delta F$ contains an alternating cycle which includes both $e$ and $e^{\prime}$.

Suppose now that the theorem is true whenever $|P| \leqq k$ for some integer $k>1$, and let $|P|=k+1$. Let $e_{k}$ be the edge of $P-\left\{e^{\prime}\right\}$ that has an end in common with $e^{\prime}$. Let $A_{1}$ be an alternating cycle containing $e$ and $e_{k}$ and let $A_{2}$ be an alternating cycle containing $e_{k}$ and $e^{\prime} ; A_{1}$ and $A_{2}$ exist by the induction hypothesis. Let $F_{0}$ and $F_{1}$ be the 1-factors satisfying $A_{1}=F_{0} \Delta F_{1}$, where $e_{k} \in F_{0}$. If $e^{\prime} \in F_{1}$, then $e^{\prime} \in A_{1}$ and we are done; consequently suppose $e^{\prime} \notin F_{1}$. Thus there is a 1 -factor $F_{2}$ of $G$ suchl that $F_{2} \neq F_{0}, F_{2} \neq F_{1}$ and $e^{\prime} \in F_{2}$. Consequently we may choose $A_{2}=F_{0} \Delta F_{2}$.

Now let $H$ be the graph with $V(H)=V\left(A_{1}\right) \cup V\left(A_{2}\right)$ and $E(H)=A_{1} \cup A_{2}$. Thus $F_{0} \cap E(H)$ is clearly a 1-factor of $H$, and so $F_{0} \cap(E(G)-E(H))$ is a 1-factor of $G-V(H) . A_{1}$ is clearly an alternating cycle of $H$ since it is of even length and $F_{0} \cap\left(A_{2}-A_{1}\right)$ is a 1 -factor of $H-V\left(A_{1}\right)$. Similarly $A_{2}$ is an alternating cycle of $H$. Since $E(H)=A_{1} \cup A_{2}$ and $H$ is connected, by the lemma there is an alternating cycle $A$ of $H$ containing $e$ and $e^{\prime}$. Hence $H-V(A)$ has a 1-factor $F$. Thus $F \cup\left(F_{0} \cap(E(G)-E(H))\right)$ is a 1 -factor of $G-V(A)$, so that $A$ is an alternating cycle of $G$. The theorem is proved.

\section{Reference}

[1] M. Behzad, and G. Chartrand; Introduction to the Theory of Graphs (Allyn \& Bacon, Boston, 1971).

Department of Mathematics and Computer Science Royal Melbourne Institute of Technology, Victoria, 3000, Australia 